\title{
THE MOST COMPACT SCISSION CONFIGURATION OF FRAGMENTS FROM LOW ENERGY FISSION OF ${ }^{234} \mathrm{U}$ AND ${ }^{236} \mathrm{U}$
}

\author{
Justo A. Rojas T. ${ }^{\text {a, }}{ }^{*}$, Modesto Montoya ${ }^{\text {a, }}$ \\ ${ }^{a}$ Instituto Peruano de Energía Nuclear, Av. Canadá 1470, Lima - 41, Perú. \\ ${ }^{b}$ Facultad de Ciencias Físicas, Universidad Nacional Mayor de San Marcos Ap. Postal 14-0149, Lima 14, Perú. \\ ${ }^{c}$ Facultad de Ciencias, Universidad Nacional de Ingeniería, Av. Túpac Amaru, 210, Apartado 31-139, Lima, Perú.
}

(Recibido Junio 12, 2009; Aceptado Octubre 02, 2009)

\begin{abstract}
Using a time of flight technique, the maximal values of the kinetic energy as a function of the primary mass of fragments from low energy fission of ${ }^{234} \mathrm{U}$ and ${ }^{236} \mathrm{U}$ were measured by Signarbieux et al. From calculations of scission configurations, one can conclude that, for those two fissioning systems, the maximal values of the total kinetic energy corresponding to fragmentations $\left({ }_{42} \mathrm{Mo}_{62},{ }_{50} \mathrm{Sn}_{80}\right)$ and $\left({ }_{42} \mathrm{Mo}_{64},{ }_{50} \mathrm{Sn}_{80}\right)$ respectively, are equal to the available energy, and that their scission configurations are composed of a spherical heavy fragment and a prolate light fragment, both in their ground state.
\end{abstract}

PACS: 25.70.-z; 25.85.-w; 24.75.+i

Keywords: Low energy fission; ${ }^{234} \mathrm{U} ;{ }^{236} \mathrm{U}$; fragment kinetic energy; cold fission.

\section{Resumen}

Usando una técnica de tiempo de vuelo, Signarbieux et al. midieron el valor máximo de la energía cinética total en función de la masa primaria de los fragmentos de la fisión de baja energía de ${ }^{234} \mathrm{U}$ y ${ }^{236} \mathrm{U}$. De los cálculos de las configuraciones de escisión, puede concluirse que, para esos dos sistemas físiles, el valor máximo de la energía cinética corresponde a las fragmentaciones $\left({ }^{104} \mathrm{Mo},{ }^{130} \mathrm{Sn}\right)$ y $\left({ }^{106} \mathrm{Mo},{ }^{130} \mathrm{Sn}\right)$, respectivamente, son iguales a los valores disponibles de energía, y sus configuraciones de escisión están compuestas por un fragmento pesado esférico y un fragmento liviano prolato, ambos en sus estados fundamentales.

Palabras claves: Fisión a baja energía; ${ }^{234} \mathrm{U} ;{ }^{236} \mathrm{U}$; energía cinética de fragmentos; fisión fría.

\section{Introduction}

One of the most studied quantities to understand the fission process are the fragment mass and kinetic energy distributions, which are very closely related to the topological features in the multi-dimensional potential energy surface [1]. Structures in the distributions of mass and kinetic energy may be interpreted by shell effects on potential energy of the fissioning system, determined by the Strutinsky prescription and discussed by Dickmann [2] and Wilkins [3].

In order to investigate the fragments with very low excitation energy, using the time of flight method, Signarbieux et al. [4] measured the fragment mass distribution for high values of fragment kinetic energy. Because in that kinetic energy region there is no neutron emission, the time of flight technique permits separate neighboring fragment masses. In this work one calculates the deformations of those fragments which must correspond to the most compact scission configurations, i.e. to the highest values of Coulomb interaction energy between the two fragments.

\section{The most compact scission configurations}

In the process of thermal neutron induced fission of ${ }^{233} \mathrm{U}$, the compound nucleus ${ }^{234} \mathrm{U}$ with excitation energy equal to neutron separation energy $\left(B_{\mathrm{n}}\right)$ is formed first. Then, this nucleus splits into two complementary light and heavy fragments having $A_{L}$ and $A_{H}$ as mass numbers, and $E_{L}$ and $E_{H}$ as kinetic energies, respectively.

The Q-value of this reaction is given by the relation

$Q=M(92,234)-M\left(Z_{L}, A_{L}\right)-M\left(Z_{H}, A_{H}\right)$,

where $M(Z, A)$ is the mass of nucleus with $Z$ and $A$ as proton number and mass number, respectively.

\footnotetext{
*Correspondig author.e-mail: jrojast@unmsm.edu.pe
} 

be

The balance energy at scission configuration will

$$
Q+B_{n}=T K E_{O}+C E+T D E+T X E
$$

where $T K E_{0}$ is the pre-scission total kinetic energy; $C E$ is the Coulomb interaction energy between fragments;

$$
T D E=D E_{L}+D E_{H}
$$

is the total deformation energy, where $D E_{L}$ and $D E_{H}$ are the deformation energies of the light and heavy fragments respectively; and

$$
T X E=X E_{L}+X E_{H}
$$

is the total intrinsic excitation energy, where $X E_{L}$ and $X E_{H}$ are the intrinsic light and heavy fragments excitation energies, respectively.

If there is no neutron emission, the light and heavy fragments reach the detectors with their primary kinetic energies equal to $K E_{L}$ and $K E_{H}$, respectively. The total kinetic energy of the primary fragments will be

$$
T K E=T K E_{0}+C E=Q+B_{n}-T D E-T X E
$$

The maximal value of total kinetic energy is reached when the sum of TDE and TXE is minimal, i.e.

$$
T K E_{\max }=Q+B_{n}-(T D E+T X E)_{\min } .
$$

The most compact scission configuration is defined as that corresponding to

$$
T D E_{\min }=0, T X E_{\min }=0 \text { and } T K E_{0}=0 .
$$

In this case, from Eq. (6) one obtains the relations

$T K E_{\max }=C E_{\max }=Q+B_{n}$

Not always this situation is possible to occur. Nevertheless we can assume that for each mass fragmentation the maximal value of total kinetic energy is obtained for similar condition, i.e. $T K E_{0}=0, T X E=0$ and $T D E=T D E_{\min }$.

\section{Deformation energy}

The total energy (U) of a composed of nucleons, is calculated using the Strutinsky method [5]. The total energy of a nucleus is calculated at first approximation by a liquid drop model type $(\tilde{W})$, using the mass formula of Myers and Swiatecki [6].

The shell correction $(\delta U)$ is calculated by the Strutinsky's method applied by Quentin et al. [7], using Nilsson Hamiltonian [8]:

$$
V_{\text {corr }}=-\kappa\left\{\hat{l} \cdot \hat{s}+\mu\left(\hat{l}^{2}-\left\langle l^{2}\right\rangle_{N}\right)\right\}
$$

where $K$ and $\mu$ are the Nilsson's constants.

The pairing correction is calculated using the BCS method [9]. Then, the relation for the total energy of the nucleus $(Z, N)$ is given by:

$$
D E=\tilde{W}(Z, N, \varepsilon)-\tilde{W}_{S}(Z, N)+\delta U_{N}+\delta U_{Z}+\delta P_{N}+\delta P_{Z}
$$

where $\widetilde{W}(Z, N, \varepsilon)$ is the energy of a nucleus $(Z, N)$ having deformation $\varepsilon$, and $\widetilde{W}_{S}(Z, N)$ the energy in its spherical shape.

The constant of the harmonic oscillator was the suggested by Nilsson [8]:

$\hbar \omega_{0}=41 A^{1 / 3}$.

As one said, the total kinetic energy of the fragments is close to the available energy for light and heavy complementary fragments with masses around $A=104$ and $A=132$, respectively. Let us relate this result to the deformation of nuclei in this mass neighborhood.

The energy of nucleus ${ }^{106-108}$ Mo as a function of their corresponding deformations $(\varepsilon)$ are presented on Fig. 1. The assumed Nilsson's constants [7] for these nuclei are

$\kappa_{N}=0.678, \kappa_{P}=0.07, \mu_{N}=0.33$ and $\mu_{P}=0.35$.

As we can see these nuclei have a prolate shape with to $\varepsilon=0.3$ in their ground state. If the fragment deformation changes from $\varepsilon=0$ to $\varepsilon=0.3$ the deformation energy will decreases by about $2 \mathrm{MeV}$, while a change from $\varepsilon=0.3$ to $\varepsilon=0.4$ increases of deformation energy by $4 \mathrm{MeV}$. This result suggests that these nuclei are prolate and soft between $\varepsilon=0$ to $\varepsilon=0.3$ and became stiff for higher prolate deformations.

The deformation energy as a function deformation for nuclei ${ }^{130-132} \mathrm{Sn}$ are presented in Fig.2. The assumed Nilsson's constants for these nuclei are

$\mathrm{K}_{\mathrm{n}}=0.0635, \mathrm{~K}_{\mathrm{p}}=0.067$

$\mu_{\mathrm{N}}=0.43, \mu_{\mathrm{p}}=0.54$ 
One can see that ${ }^{130} \mathrm{Sn}$ is softer than ${ }^{132} \mathrm{Sn}$. For a deformation from $\varepsilon=0$ to $\varepsilon=0.2$, the nucleus ${ }^{130} \mathrm{Sn}$ spends around $5 \mathrm{MeV}$ while the nucleus ${ }^{132} \mathrm{Sn}$, for the same deformation, spends $10 \mathrm{MeV}$. The neutron number $N=82$ and proton numbers around $Z=50$ correspond to spherical hard nuclei.

The above characteristics of light fragments, corresponding to masses from $A=100$ to $A=106$, and their complementary fragments, corresponding to masses from $A=130$ to $A=132$, make possible that the maximal values of their total kinetic energy of complementary fragments $T K E$ be close to the available energy.

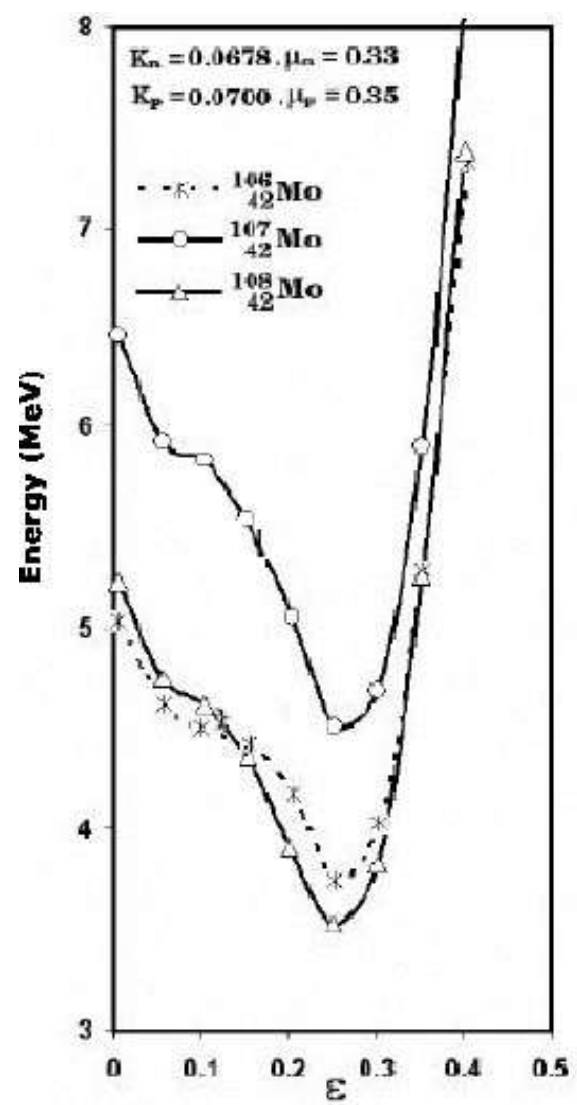

Fig. 1. Deformation energy for nuclei ${ }^{106-108} \mathrm{Mo}$ calculated by a liquid drop model with pairing and shell correction [6]. See text

For the case of ${ }^{233} U\left(n_{t h}, f\right)$, the total kinetic energy of the pair $\left({ }_{42} \mathrm{Mo}_{62},{ }_{50} \mathrm{Sn}_{80}\right)$ is almost equal to the available energy. This result means that the corresponding scission configuration is composed by fragments in their ground state. In Fig. 3 we can see the equipotential energies of the scission configurations composed by those fragments given by the relation

$$
V=C E+D E_{H}+D E_{L}
$$

where $D E_{H}$ and $D E_{L}$ are the heavy and light fragment deformation energy, respectively, calculated using the Nilsson model [8] and $C E$ is the Coulomb interaction energy between the two fragments separated by $2 \mathrm{fm}$. On this curve one obtains that for $\varepsilon_{H}=0$ and $\varepsilon_{L}=0.3$ the Coulomb energy is equal to the available energy of $204 \mathrm{MeV}$.

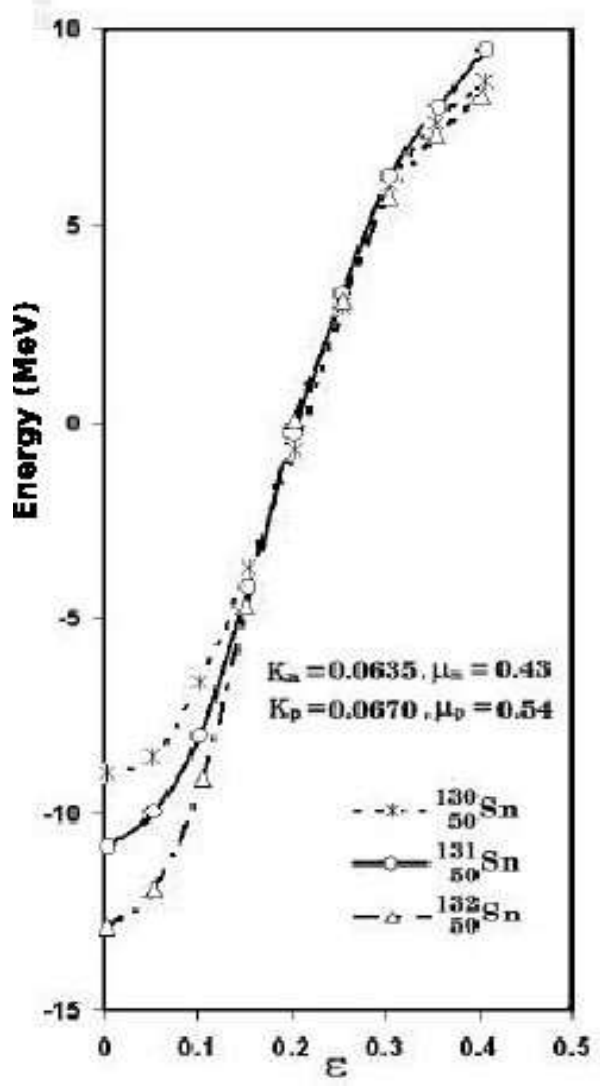

Fig. 2. Deformation energy for nuclei ${ }^{130-132} \mathrm{Sn}$ calculated by a liquid drop model with pairing and shell correction [6]. See text.

The results are similar to complementary fragments corresponding to the deformed transitional nuclei with $A_{L}$ between 100 and 106 ( $N$ between $60 \mathrm{y}$ 64 ) and to the spherical nuclei with $A_{H}$ around 132 ( $\mathrm{Z}$ $=50$ and $\mathrm{N}=82$ ).

For the complementary fragments ${ }^{104} \mathrm{Mo}$ and ${ }^{130} \mathrm{Sn}$ the maximal value of $\mathrm{CE}$ corresponds to ground state nuclei or close to that. This case is unique. Other configurations will need deformation energy, which will be higher for the harder nuclei.

In Fig. 3 the deformation energy is presented for the spherical nuclei ${ }^{130} \mathrm{Se},{ }^{131} \mathrm{Se}$ and ${ }^{132} \mathrm{Se}$, respectively. We can see that the double magic nucleus ${ }^{132}$ Se needs $2 \mathrm{MeV}$ more than ${ }^{130} \mathrm{Se}$ for going from the spherical state $\varepsilon=0$ to the slightly deformed $\varepsilon=0.05$. The fact that ${ }^{132} \mathrm{Se}$ is no so hard as ${ }^{132} \mathrm{Se}$ explains why the highest values of Coulomb 
interaction energy corresponds to values close to the available energy for ${ }^{233} \mathrm{U}\left(\mathrm{n}_{\mathrm{th}}, \mathrm{f}\right)$ as well as for ${ }^{235} \mathrm{U}\left(\mathrm{n}_{\mathrm{th}}, \mathrm{f}\right)$.

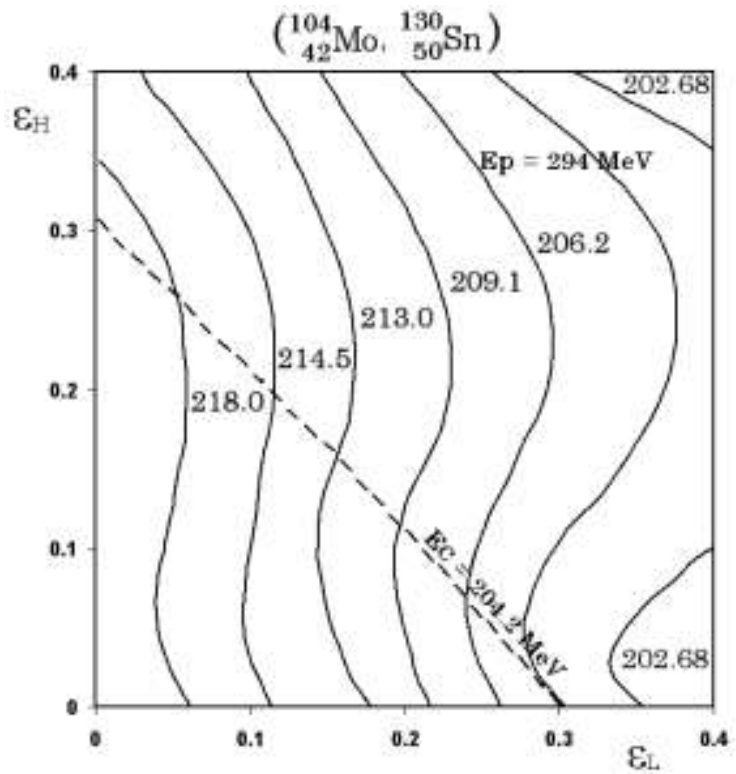

Fig.3. Equipotential curves for scission configuration of fragments ${ }^{104} \mathrm{Mo},{ }^{130} \mathrm{Sn}$ as a function of their deformation. $\varepsilon_{L}$ and $\varepsilon_{H}$ are the light and heavy fragment deformation.

\section{Conclusions}

From the calculation of scission configurations by thermal neutron induced fission of ${ }^{233} \mathrm{U}$ and ${ }^{235} \mathrm{U}$, respectively, one can conclude that the highest value of Coulomb interaction energy between complementary fragments corresponds to fragmentations $\left({ }^{104} \mathrm{Mo},{ }^{130} \mathrm{Sn}\right)$ and $\left({ }^{106} \mathrm{Mo},{ }^{130} \mathrm{Sn}\right)$, respectively. For both cases the calculated maximal values of Coulomb interaction energy values are equal to the available energy of the reaction for spherical $\left(\varepsilon_{H}=0\right)$ heavy fragments and prolate $\left(\varepsilon_{L}=\right.$ $0.3)$ complementary light fragments, which correspond to their ground states. Moreover the light fragments are soft between $\varepsilon_{L}=0$ and $\varepsilon_{L}=0.3$ and harder if they go to more prolate shapes; while the spherical heavy fragment ${ }^{130} \mathrm{Sn}$ is no as hard as ${ }^{132} \mathrm{Sn}$. The calculated maximal value of Coulomb interaction energy is equal to the measured maximal value of total kinetic energy of fragments. The prescission kinetic energy and intrinsic excitation energy of the fragments are assumed to be null. These results suggest that the fission process take time to explore all energetically permitted scission configurations

\section{6.- References}

[1].P. Möller, D. G. Madland, A. J. Sierk, A. Iwamoto, Nature 409(2001)785

[2].F. Dickmann and K. Dietrich, Nucl. Phys. A129(1969)241.

[3].B. D.Wilkins, E.P. Steinberg and R.R. Chasman, Phys. Rev. C14(1976)1832.

[4].C. Signarbieux, M. Montoya, M. Ribrag, C. Mazur, C. Guet, P. Perrin and M. Maurel, J. Phys. Lett. (Paris), 42(1976)L-437 - L-440..

[5].V. M. Strutinsky, Nucl. Phys., A95 (1967) 420

[6].W. D. Myers and W.S. Swiatecky, Nucl. Phys. 81(1966)1.

[7].P. Quentin and R. Babinet, Nucl. Phys. A159 (1970)365-384 and references therein.

[8].C. G. Nilsson, Mat. Fys. Medd. Dan. Vid. SelsK 29 (1955) n.16.

[9].S.T. Belyaev, Mat. Fys. Medd. Vid. Selsk, 31(1959) n.1. 